\title{
Propuesta de construcción de competencias de innovación en la formación de ingenieros en el contexto de la industria 4.0 y los objetivos de desarrollo sostenible (ODS)
}

\author{
Felipe C. Muñoz-La Rivera ${ }^{1,2}$, Pamela Hermosilla ${ }^{3}$, Jean Delgadillo1, y Dayan Echeverría ${ }^{4}$ \\ (1) Escuela de Ingeniería Civil, Pontificia Universidad Católica de Valparaíso, Valparaíso, Chile. \\ (correo-e: felipe.munoz@pucv.cl; jean.delgadillo@pucv.cl) \\ (2) Escuela de Caminos, Canales y Puertos, Universidad Politécnica De Cataluña, Barcelona, España. \\ (3) Escuela de Ingeniería Informática, Pontificia Universidad Católica de Valparaíso, Valparaíso, Chile. \\ (correo-e: pamela.hermosilla@pucv.cl) \\ (4) Valparaíso Makerspace, Pontificia Universidad Católica de Valparaíso, Valparaíso, Chile. \\ (correo-e: dayan.echeverria@pucv.cl)
}

Recibido Jul. 31, 2020; Aceptado Sep. 28, 2020; Versión final Nov. 27, 2020, Publicado Abr. 2021

\begin{abstract}
Resumen
El objetivo de investigación es identificar las habilidades de innovación que los futuros ingenieros deben adquirir en respuesta a los desafíos de la ingeniería del siglo XXI, los requisitos de la actual industria 4.0 y los objetivos de desarrollo sostenible (ODS). El método está organizado en dos etapas: 1) identificación de las características de la innovación y 2) construcción de habilidades de innovación para los estudiantes de ingeniería. Esta investigación esta basada en una revisión de literatura en Web of Science y Scopus desde el año 2000 hasta el presente. Se plantea un modelo que integra los ODSs en el contexto de la industria 4.0 y las habilidades de innovación en el ámbito de ingeniería. Se concluye que el modelo descrito aquí es una representación concreta que permite integrar los conceptos relacionados con los objetivos de desarrollo sostenible y la industria 4.0 para incorporarlos en el ámbito de la educación universitaria.
\end{abstract}

\section{Proposal for the construction of innovation skills in engineering education in the context of industry 4.0 and sustainable development goals (SDG)}

\begin{abstract}
The main objective of the present research study is to identify innovation skills that future engineers should acquire to meet the challenges of XXI century engineering, the increasing demand requirements of the industry 4.0, and sustainable development goals (SDG). The methodology is divided in two stages: 1) to identify innovation traits and 2) to build innovation abilities for engineering students. A literature search is performed on Web of Science and Scopus from the year 2000 to present. A model is proposed for SDGs by using an industry 4.0 context and engineering innovation abilities. It is concluded that the model described here is a concrete representation that allows integrating sustainable development goals and industry 4.0 concepts to incorporate them in higher-level education.
\end{abstract}

Keywords: innovation skills; engineering education; industry 4.0; sustainable development 


\section{INTRODUCCIÓN}

La industria 4.0 representa un nuevo paradigma de digitalización y automatización de los procesos de fabrica-ción. Esta propone la implementación de entornos de trabajo inteligentes, ampliamente interconectados, automáticos, robotizados y sistematizados a alto nivel (Oesterreich \& Teuteberg, 2016). Estos cambios respecto a la industria tradicional demandarán a los trabajadores nuevas habilidades y competencias. En el área de la ingeniería, se requerirán profesionales con amplias habilidades digitales, aprendizajes ágiles, trabajo en equipo, resolución de problemas, comunicación efectiva e innovación, que sepan responder a los nuevos funcionamientos de las plantas de producción y se integren exitosamente a las dinámicas de trabajo digitales y automáticas (Rejikumar et al., 2019).

En el contexto del siglo XXI, y en respuesta al funcionamiento de las industrias y la economía, las Naciones Unidas han establecido una serie de acuerdos para alinear las políticas de desarrollo global en un marco común que permita resguardar la sostenibilidad global (Morton et al., 2019). Los objetivos de desarrollo sostenible (ODS) corresponden a 17 objetivos dentro de un acuerdo universal que busca abordar la evidencia empírica y científica de que el mundo necesita un enfoque más sostenible en sus acciones de desarrollo (Naciones Unidas, 2015). En este contexto, se destaca la importancia de la innovación para responder exitosamente a los desafíos profesionales de la Industria 4.0, alineados con los compromisos de los objetivos de desarrollo sostenible. Profesionales dinámicos e innovadores serán clave para la integración de los sistemas de producción, la eficiencia de la interacción humano - sistemas automáticos, y la solución de nuevos problemas que surgirán asociados a estos sistemas de producción.

Estas características de innovación incorporadas en el contexto de la industria 4.0 y los ODS deben considerar competencias blandas y competencias tecnológicas, desde la perspectiva de gestión e integración con personas, hasta el conocimiento y dominio de herramientas digitales (Cabaña et al., 2019). La industria de la ingeniería global ha establecido diferentes características para el ingeniero innovador, las que tratan de adaptabilidad, búsqueda constante de soluciones, experimentador e integrador, con amplios conocimientos, curioso y comunicador, responsable, persistente y apasionado, colaborador e integrador, creativo, visionario, desafiante, líder, con inteligencia de negocios y focalizado en el usuario (Hermosilla et al., 2020). Si bien, existe evidencia de las demandas de la industria para sus profesionales ingenieros ante estos nuevos desafíos, que se tornan hoy muy necesarios en industrias con bajos niveles de innovación y productividad, como es el caso de la industria de la construcción, no se ha estudiado ampliamente la visión desde los entornos formativos, respecto a la formación de estas competencias en los estudiantes universitarios de ingeniería. Particularmente no se han establecido mecanismos para definir las competencias a formar en los futuros ingenieros, para dar respuesta a los ODS y la Industria 4.0 (Liao et al., 2017). Adaptando la estructura clásica de generación de competencias educacionales, esta investigación presenta un flujo para la construcción de competencias de innovación en ingeniería, integrando los requerimientos de la industria 4.0 y el contexto y desafíos de los objetivos de desarrollo sostenible.

La integración de los distintos aspectos mencionados anteriormente, se refleja en el ámbito educacional en el concepto de competencia, relacionada con el perfil de egreso de los estudiantes aludiendo al conocimiento, habilidades y actitudes que los estudiantes requieren en el contexto de la sociedad actual, enfocada a la industria 4.0 y los ODS (Anderson, 2018). Bajo esta perspectiva fue necesario indagar en la los fundamentos de modelos de educación basada en competencia (Cruz, 2017), así como también de los lineamientos que miden y verifican la calidad de los proceses formativos (ABET, 2020).

\section{METODOLOGÍA}

El método de investigación está organizado en dos etapas: (1) Identificación de las características de la innovación para los estudiantes de ingeniería y (2) Construcción de habilidades de innovación para los estudiantes de ingeniería. La figura 1 muestra las etapas y especifica las herramientas y actividades de investigación, junto con los resultados de cada etapa.

En la primera etapa, se realizó un examen de la literatura para identificar los desafíos de los Objetivos de Desarrollo Sostenible (ODS) y los requisitos para las nuevas funciones de los profesionales de la Industria 4.0, junto con la identificación de las características de innovación en la ingeniería. La búsqueda se realizó en las bases de datos Web of Science y Scopus, junto con la incorporación de manuales de innovación de entidades reconocidas en el área y organizaciones de acreditación universitaria. Para identificar estas características fue necesario reconocer algunas palabras clave, las cuales fueron seleccionadas en base a su relevancia, frecuencia y tendencia de aplicación. La búsqueda se realizó en las bibliotecas Web of Science y Scopus, junto con la incorporación de manuales de innovación de entidades reconocidas en el área y organismos de acreditación universitaria. Los documentos fueron seleccionados desde el año 2000 hasta el presente. Se consideraron los temas de búsqueda: a) Objetivos de desarrollo sostenible - SDG 
(desafíos, implicaciones); b) Industria 4.0 (nuevos profesionales, desafíos); e innovación en ingeniería (características, habilidades, destrezas). Posteriormente, a partir del soporte bibliográfico, el equipo de investigación analizó y categorizó las características de la innovación para los estudiantes de ingeniería en grupos de habilidades.

En la segunda etapa, a partir de una nueva revisión bibliográfica (en las mismas colecciones ya definidas), se identificaron las características para la construcción de competencias educativas. Con esto, el equipo de investigación desarrolló un flujo de trabajo para la construcción de competencias de innovación para los estudiantes de ingeniería (en los contextos de Industria 4.0 y ODS). Este flujo fue validado por un panel de expertos, dónde se seleccionaron cinco expertos que cumplían los siguientes requisitos: a) más de 10 años de práctica en actividades de innovación, y b) experiencia como consultor, investigador o profesor universitario en temas de innovación. Sobre la base de un grupo de discusión, los expertos examinaron dicho flujo que facilita la construcción de competencias de innovación para estudiantes de ingeniería.

ETAPA HERRAMIENTAS

ACTIVIDADES

ENTREGABLES

(1)

Características

de innovación

para estudiantes

de ingeniería

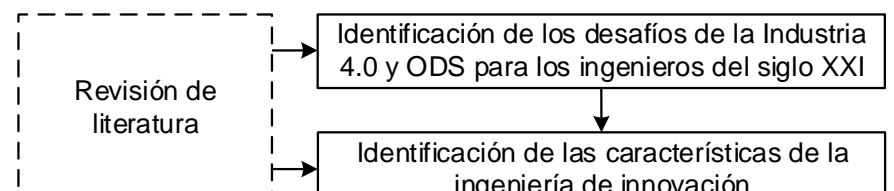

mección de

competencias

de innovación para

estudiantes de ingeniería

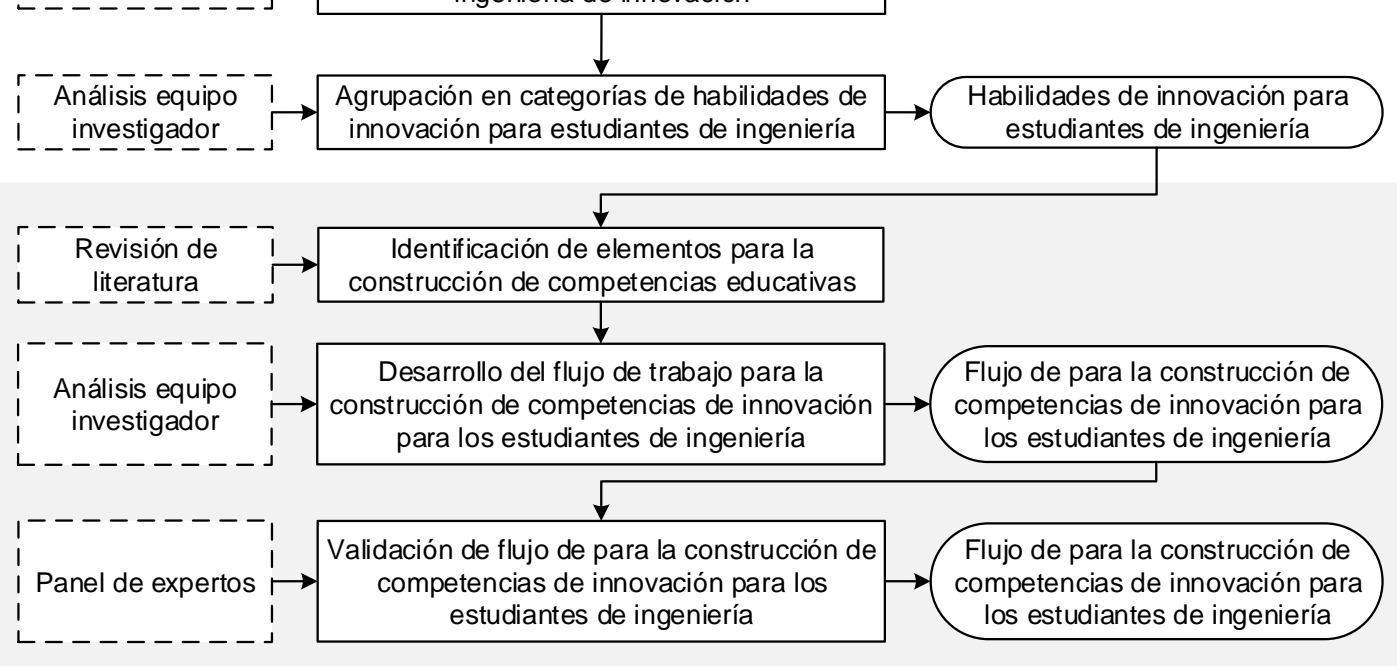

Fig. 1: Metodología de Investigación

\section{Antecedentes de la literatura}

El concepto de Industria 4.0 describe la tendencia a la digitalización y automatización de los entornos de fabricación (Oesterreich y Teuteberg, 2016). Acorde a Mohamed (2018) la industria 4.0 "es la suma de todas las innovaciones disruptivas derivadas y aplicadas en una cadena de valor para hacer frente a las tendencias en materia de digitalización, automatización, transparencia, movilidad, modularización, colaboración en red y socialización de productos y procesos". Por lo tanto, la Industria 4.0 puede implementar un entorno y proporcionar un servicio más inteligente, haciendo que los procesos se lleven a cabo perfectamente en varios sectores de la industria, teniendo una amplia gama de aplicaciones. Aunque Industria 4.0 es un término establecido en el mundo moderno de hoy, su significado y su implementación todavía está ausente en algunos casos debido a la falta de conciencia real de las personas en estos temas (Rejikumar et al., 2019). Los avances tendrán un profundo efecto en la sociedad y en los individuos, ya que se mejorará la producción, integración y manejo de los sistemas informáticos.

Uno de los grandes desafíos que las organizaciones tendrán que resolver es la capacidad técnica de sus trabajadores, ya que tendrán que ser capaces de analizar los fallos, superar los constantes cambios, aprender nuevas tareas y centrarse en la resolución de problemas. Por lo tanto, la capacitación y el desarrollo profesional continuo serán un factor crítico en el éxito de la Industria 4.0, ya que no solo cambiará las ocupaciones que los trabajadores desempeñan actualmente, sino que también modificará los marcos de habilidades (Xu et al., 2018). La innovación dinámica desempeñará un papel importante, ya que ésta brinda la oportunidad de desarrollar nuevas organizaciones y modelos empresariales, lo que conlleva un mayor grado de participación de los representantes. Además, el proceso de digitalización y virtualización asegura y 
presenta varias oportunidades para que los fabricantes desarrollen nuevos valores e impulsen la innovación. Sin embargo, hay pocos estudios en el ámbito de la educación de ingeniería y gestión, respecto a las necesidades formativas de los estudiantes universitarios que serán la fuerza de trabajo en esta dinámica revolución que se vive (Liao et al., 2017).

Por su parte, los gobiernos han asumido un papel de liderazgo y muchos de ellos han decidido iniciar un viaje evolutivo hacia la Industria 4.0 (Rejikumar et al., 2019). Por lo tanto, es de esperar que dicha transformación se produzca de acuerdo con los objetivos y metas que cada país se compromete a cumplir tanto con su propia nación como con el mundo entero. En este contexto, y amparados en el compromiso asumido por un gran número de países, las iniciativas para el desarrollo de las industrias en el siglo XXI se vinculan a los Objetivos de Desarrollo Sostenible (ODS) propuestos por las Naciones Unidas. Los ODS son un conjunto de objetivos dentro de un acuerdo universal que pretenden abordar la evidencia empírica y científica de que el mundo necesita un enfoque más sostenible (Morton et al., 2019). En consecuencia, los ODS tienen como objetivo reflejar los principios morales de que ningún país ni nadie debe quedar rezagado, siendo todos responsables de hacer realidad la visión global, y de expresar los desafíos de sostenibilidad a los que se enfrenta el mundo (Osborn et al., 2015).

Los objetivos se resumen en 17 áreas de impacto, que proporcionan un marco suficientemente sólido desde el punto de vista científico, políticamente aceptable y públicamente intuitivo. Estos objetivos son: (1) poner fin a la pobreza en todas sus formas y en todo el mundo; (2) poner fin al hambre, lograr la seguridad alimentaria y la mejora de la nutrición, y promover la agricultura sostenible; (3) garantizar una vida sana y promover el bienestar de todos a cualquier edad; (4) garantizar una educación inclusiva y equitativa de calidad, y promover oportunidades de aprendizaje permanente para todos; (5) lograr la igualdad de género y empoderar a todas las mujeres y las niñas; (6) garantizar la disponibilidad y la gestión sostenible del agua y el saneamiento para todos; (7) garantizar el acceso a una energía asequible, fiable, sostenible y moderna para todos; (8) promover el crecimiento económico sostenido, inclusivo y sostenible, el empleo pleno y productivo, y el trabajo decente para todos (9) construir infraestructuras resilientes, promover la industrialización inclusiva y sostenible, y fomentar la innovación; (10) reducir la desigualdad en los países y entre ellos; (11) lograr que las ciudades y los asentamientos humanos sean inclusivos, seguros, resilientes y sostenibles; (12) garantizar modalidades de consumo y producción sostenibles; (13) adoptar medidas urgentes para combatir el cambio climático y sus efectos; (14) conservar y utilizar sosteniblemente los océanos, los mares y los recursos marinos para el desarrollo sostenible; (15) proteger, restablecer y promover el uso sostenible de los ecosistemas terrestres, gestionar sosteniblemente los bosques, luchar contra la desertificación, detener e invertir la degradación de las tierras y detener la pérdida de biodiversidad; (16) promover sociedades pacíficas e inclusivas para el desarrollo sostenible, facilitar el acceso a la justicia para todos y construir a todos los niveles instituciones eficaces e inclusivas que rindan cuentas; y (17) fortalecer los medios de implementación y revitalizar la Alianza Mundial para el Desarrollo Sostenible (Naciones Unidas, 2015).

En estos contextos de desarrollo, será clave la innovación centrada en el uso de nuevos conocimientos para la creación y/o mejora de nuevos servicios, procesos o productos. Esta permitirá obtener resultados originales de alto valor social y/o económico, que respondan a retos de sostenibilidad y los entornos tecnológicos futuros (Sung, 2018). Con esto, las habilidades de innovación serán esenciales para la inclusión exitosa de los profesionales en la Industria 4.0, proporcionando una posibilidad de desarrollo enmarcada en los nuevos objetivos de desarrollo (Toner, 2011).

En el campo de la ingeniería, muchos autores han indicado las características que deben tener los ingenieros innovadores. En este contexto, se han identificado 20 características de innovación que necesita poseer un profesional del área de la ingeniería: debe ser adaptable y flexible (enérgico, activo para aprender, hacer y rehacer, aceptando positivamente la opinión de los demás); buscador de alternativas múltiples (capaz de buscar una mejor manera de ejecutar un proceso, diseñar o fabricar un producto); experimentador (capaz de aceptar la incertidumbre, utilizando la creación de prototipos para evaluar las opciones); integrador de conocimientos (capaz de integrar los propios conocimientos con los del equipo y técnico para construir nuevas soluciones); con conocimiento profundo (profesional educado y conocedor de una amplia gama de temas); curioso por hacer y aprender (profesional reflexivo, en constante búsqueda de nuevas ideas y soluciones); comunicador (capaz de comunicar eficazmente las ideas y persuadir a otros); responsable (capaz de tomar el control de sus actividades y supervisar un proyecto de principio a fin, respondiendo a los resultados y aceptando los posibles errores); persistente (comprometido, decidido, resistente.

Además, tiene la convicción de que logrará sus objetivos); apasionado (profesional que se apasiona por su trabajo y los objetivos que ha logrado, transmitiendo su pasión a su equipo); colaborador e integrador (capaz de colaborar con otras personas con conocimientos y habilidades específicas, para lograr con éxito 
una innovación); creativo (capaz de generar nuevas ideas o asociaciones entre ideas para producir soluciones originales); arriesgado (dispuesto a correr riesgos y fracasar); visionario (profesional con una visión y objetivos claros); desafiante (dispuesto a hacer las cosas de manera diferente, cuestionando los métodos tradicionales); líder y gestor de equipo (profesional que es un líder y gestor de equipo, facilitando e impulsando la innovación); implementador (que organiza y gestiona de manera eficiente los recursos para desarrollar una innovación); analítico (meticuloso, que examina cuidadosamente); con inteligencia de negocios (que conoce el papel de las finanzas, las ventas, las cadenas de suministro y el mercado de la innovación); y centrado en el usuario (que busca soluciones centradas en las necesidades del usuario) (Edwards-Schachter et al., 2015; Gómez et al., 2019; Hebles y Llanos-Contreras, 2019; Jarrar y Anis, 2016; Lounsbury et al., 2018; Mayhew et al., 2012; Oswald, 2015; Shuli et al., 2018).

Estas características de innovación incorporadas en el contexto de la industria 4.0 deben considerar competencias blandas y competencias tecnológicas (Radharamanan y Juang, 2012). Las primeras se enfocan en las competencias que la industria requiere para asumir cargos asociados al trabajo en equipo, liderazgo, comunicación efectiva, conocimientos del área, negociación y habilidades de negocio, orientación al servicio y al logro, pensamiento analítico, resolución de problemas, relaciones interpersonales, toma de decisiones, entre otros (Herrera et al., 2017a; Herrera et al., 2018). Por otro lado, las competencias tecnológicas se asocian a la capacidad de búsqueda de información digital y de conocimientos básicos digitales, uso de tecnologías para procesos de comunicación e interactuación de recursos digitales y creación de contenidos, programación informática, diseño y elaboración de contenidos digitales, seguridad de datos e información, y resolución de problemas técnicos (Cabaña et al., 2019).

En estos contextos, la formación en innovación para los estudiantes de ingeniería es clave (Jiménez-Medina y Rojas-Arenas, 2019) y debe estar alineada con los desafíos que plantea la Industria 4.0 y los ODS (Psacharopoulos y Schlotter, 2010) Así lo indican los organismos internacionales de acreditación, que señalan que la capacidad de innovación y la creatividad deben tenerse en cuenta en toda la formación de los futuros ingenieros (ABET, 2020). Las universidades deben responder a estos requisitos y establecer una estructura para guiar la enseñanza de estos temas. Para ello, las carreras de ingeniería requieren la incorporación de la formación de habilidades de innovación, que deben ser construidas de acuerdo a las metodologías educativas establecidas (Herrera et al., 2017b; Stock, 2018).

Una competencia en educación es un conjunto de comportamientos sociales, afectivos y habilidades cognitivas, psicológicas, sensoriales y motoras (Espada et. al, 2018) que permiten llevar a cabo adecuadamente un papel, una actuación, una actividad o una tarea (Wiek et al., 2011). Existen diversos estudios para la denominación de una competencia (Passow y Passow, 2017) en el contexto de la educación superior, de los cuales es posible identificar 4 aspectos transversales: (1) Dominio de acción; (2) Saber; (3) Saber Ser; y (4) Saber Hacer.

El (1) Dominio de acción indica el nivel de complejidad que se posee sobre un objeto de estudio, aludiendo al grado de apropiación del constructo conceptual. En este contexto, la taxonomía de Bloom y su actualización por Anderson, establece modelos jerárquicos para clasificar los objetivos de aprendizaje en diferentes niveles de complejidad, que dependen de la adquisición de conocimientos y diferentes habilidades (Anderson y Krathwohl, 2001). Por otro lado, el (2) Saber: cuerpo de conocimiento que caracteriza a la disciplina. Pueden ser conocimientos, desarrollo de modelos, aplicación de técnicas, utilización de instrumentos, uso de recursos u otros productos profesionales, entre otros. El (3) Saber Ser: demuestra la parte actitudinal en la aplicación de conocimientos y habilidades (saber), lo que da al profesional un rasgo distinto en la aplicación y por ende una significancia distintiva en los resultados obtenidos. Debe reflejar una denominación transversal de su formación, más allá de su propia área disciplinar. Muestra actitudes y valores que permiten identificar un sello en la formación. Por último, el (4) Saber Hacer: comprende por un lado el espacio (contexto) donde se inserta el profesional para desarrollar su actuación. Pueden ser contextos mundiales (instituciones, sector público y/o privado) o ámbitos específicos, como también referencia la calidad (condición de desempeño), reflejada por la posición que el profesional toma cuando se enfrenta a una situación determinada (Solesvik, 2019). La Figura 2 muestra el esquema de interacción de elementos mencionados, en la construcción de una competencia.

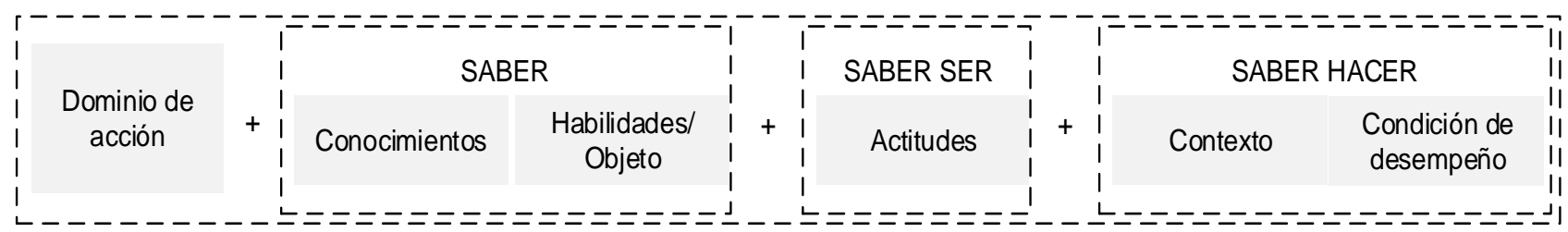

Fig. 2: Esquema de Interacción de elementos para la construcción de una competencia 
Objetivos de Desarrollo Sostenible

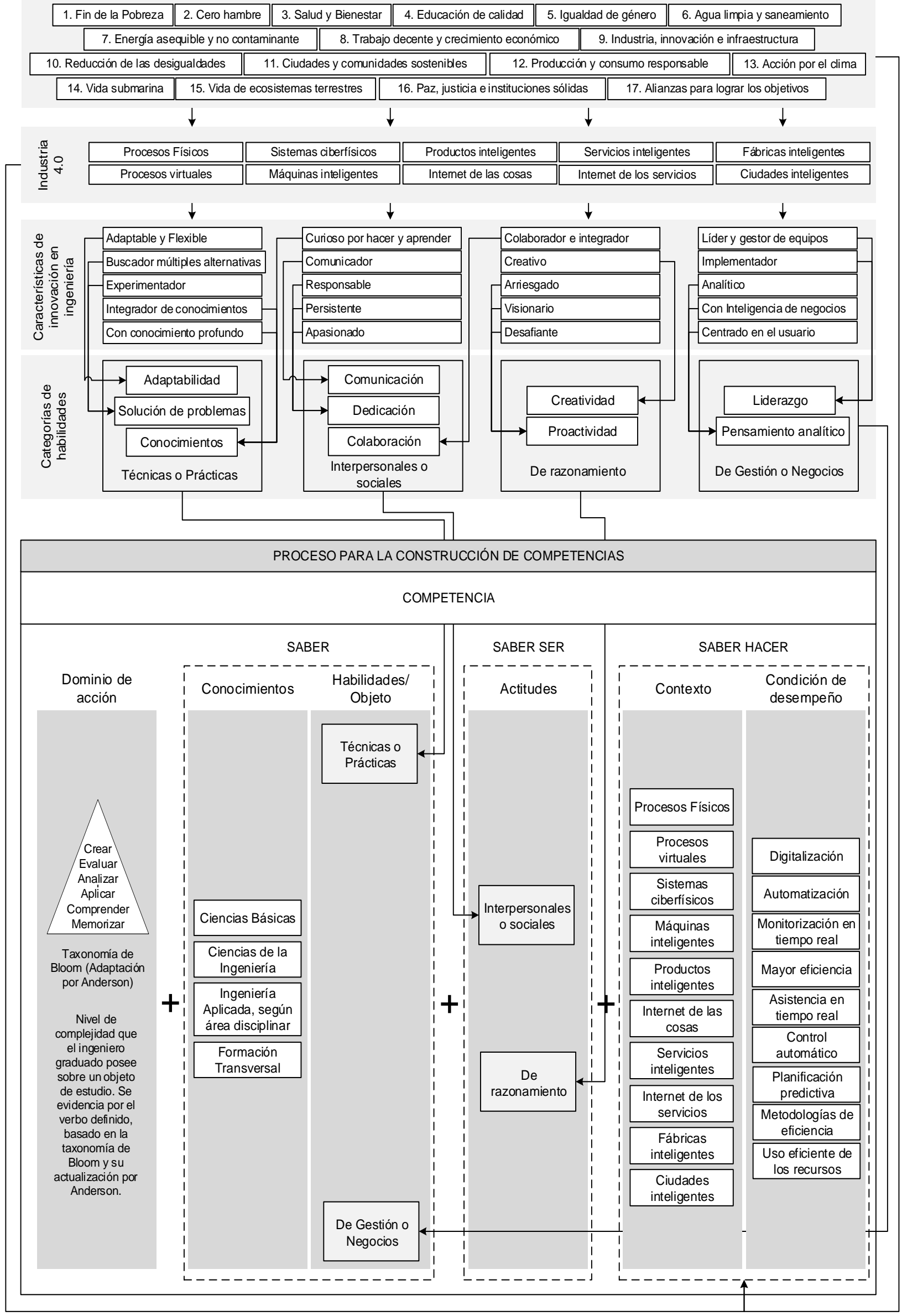

Fig. 3: Modelo para la construcción de competencias de innovación en ingeniería en el contexto de la Industria 4.0 y ODS. 


\section{Modelo para la construcción de competencias}

A partir de los antecedentes descritos en la revisión de literatura, se plantea en esta sección un modelo que integra por un lado los ODS en el contexto de la Industria 4.0 y, las habilidades de innovación en el ámbito de ingeniería y el esquema de integración de elementos en la construcción de una competencia. La Figura 3 muestra el flujo propuesto para la construcción de competencias de innovación en ingeniería en el contexto de la Industria 4.0 y los objetivos de desarrollo sostenible. Este puede ser aplicado a cual área disciplinar de ingeniería, dado que la Industria 4.0 y el contexto de los desafíos de los ODS impacta a todas los sectores productivos.

El modelo propuesto para la construcción de competencias de innovación en ingeniería contiene, en la parte superior los 17 objetivos de desarrollo sostenible, los cuales han sido identificados de los objetivos establecidos por las Naciones Unidas, aspectos que hoy en día son fundamentales es en la formación de profesionales, con miras a su futuro desempeño profesional y en la cual el área de ingeniería tiene mucho que aportar. Por otro lado, se presentan los elementos relevantes para la industria 4.0 según lo analizado en la revisión de literatura, considerando entre los aspectos más significativos la virtualización y digitalización, interconexión de sistemas físicos y digitales, la incorporación de sistemas inteligentes en las industrias, y el rol que los nuevos profesionales realizarán en su quehacer laboral, bajo estos nuevos paradigmas de trabajo.

Junto con lo anterior, la indagación realizada para conocer y especificar las características de innovación en el proceso de formación, permitió establecer las 20 características de profesionales innovadores en ingeniería, las que han confluido en 10 habilidades agrupadas en 4 categorías (1) Técnicas o Prácticas; (2) Interpersonales o sociales; (3) De razonamiento; y (4) De gestión o negocios. En el proceso de selección de estas características se han considerado implícitamente los aspectos descritos en la sección ya señalada (ODS e Industria 4.0). De esto modo, teniendo como referencia lo indicado, se integran en el modelo propuesto los elementos que interactúan en la denominación de una competencia, siendo posible conectar los aspectos del esquema de la Figura 2, donde "el saber" y "el saber ser" se relacionan con las categorías identificadas a partir de las características de innovación para los ingenieros, mientras que "el saber hacer" se relaciona con los elementos de la industria 4.0 y los ODS, reflejándose su impacto en el contexto y en la condición de desempeño.

Es importante mencionar que la aplicación de este modelo, debe considerar algunos lineamientos generales a definir previamente, los cuales son fundamentales para una adecuada implantación. No obstante, si bien el enfoque de este estudio es el planeamiento del modelo en sí, se entregan a continuación algunas consideraciones a tener en cuenta al momento de instaurarlo: (1) La identificación de los objetivos organizacionales deben ser considerados como base para la interrelación de los elementos del modelo, de tal modo que éstos pueden tener distinto énfasis dado la naturaleza y el impacto en la sociedad del ámbito de la organización donde se aplique; (2) Considerar el timing adecuado para el cumplimento de los objetivos propuestos, es decir la previsión del tiempo correspondiente para la realización de una tarea o acción; (3) Identificar las capacidades instaladas y las requeridas para el cumplimiento de los objetivos organizacionales, a modo de poder identificar aquellos puntos fuertes y los que requieren un mayor desarrollo y apropiación por parte de las personas; y (4) Identificar los indicadores asociados al cumpliendo de los objetivos, relacionando adecamente recurso y metas asociados, las que debieran considera un monitoreo y plan de acción para el cumplimento de éstos.

\section{CONCLUSIONES}

El modelo descrito en este estudio es una representación concreta que permite integrar los conceptos relacionados con los objetivos de desarrollo sostenible (Mohamed, 2018) y la industria 4.0 para incorporarlos en el ámbito de la educación universitaria (Jiménez-Medina y Rojas-Arenas, 2019), a través de la identificación de aquellas competencias de innovación que apuntan a fortalecer el proceso de formación de estudiantes en ingeniería. En las referencias citadas en este estudio es posible evidenciar las iniciativas y experiencias actuales realizadas en este ámbito, las que sustentan el modelo propuesto. De este modo, los rediseños o ajustes curriculares en el área de la ingeniería, debieran considerar los lineamientos del entorno indagado, al momento de definir los perfiles de egreso y las correspondientes competencias que darán cuenta de éste, de manera tal que los profesionales tengan los conocimientos, habilidades y actitudes requeridas para enfrentar los desafíos y necesidades de la sociedad actual enmarcadas en la innovación y en la preocupación por el entorno como parte de quehacer laboral.

De los estudios revisados, fue posible indagar en varios aspectos de los abordados en este artículo de manera más bien independiente entre sí, no así la integración de elementos de la industria 4.0, los ODS, bajo el enfoque de competencia en el proceso de formación de profesionales. De tal modo, lo propuesto en 
este estudio, puede considerarse un punto de partida inicial como apoyo al proceso de rediseño curricular, y el establecimiento de las competencias que responden al perfil de egreso declarado, no obstante, el éxito de la incorporación de estas competencias en el proceso formativo requiere revisar de manera más exhaustiva la forma de abordar adecuadamente el seguimiento y evaluación de estas competencias, a lo largo del avanece curricular del plan de estudio en el cual se insertan, lo que puede considerarse un interesante y desafiante trabajo futuro.

\section{AGRADECIMIENTOS}

Esta investigación se llevó a cabo gracias a los aportes del Proyecto CORFO 14ENI2-26905 Ingeniería 2030PUCV. Además, los autores agradecen al Collaborative Group of Engineering Education (CGEE) de la Pontificia Universidad Católica de Valparaíso. Muñoz La Rivera reconoce a CONICYT - PCHA/International Doctorate/2019-72200306 por financiar sus estudios de doctorado.

\section{REFERENCIAS}

ABET, Criteria for accrediting Engineering Programs (2020).

Anderson, L. y Krathwohl, E.A., Taxonomy for Learning, Teaching, and Assessing: A Revision of Bloom's Taxonomy of Educational Objectives, New York: Longman (2001).

Anderson, L., Competency-based education: Recent policy trends, The Journal of Competency-based Education, 3(1), $1-5$ (2018).

Cabaña, A., Galbusera, L. y Fornari, J., Industria 4.0: Competencias en carreras de ingeniería, doi: 10.33414/ajea.1.594.2019, Jornadas para Jóvenes Investigadores Tecnológicos, 1, 1-10 (2019).

Edwards, M., García, A., Sánchez, M., Quesada, H. y Amara, N., Disentangling competences: Interrelationships on creativity, innovation and entrepreneurship, doi: 10.1016/j.tsc.2014.11.006, Think. Skills Creativity, (2015).

Espada, J.S., López, S.M., Bel, P., Lejarriaga, G., Educación y formación en emprendimiento social: características y creación de valor social sostenible en proyectos de emprendimiento social. Revista de Estudios Cooperativos, 129, 1638 (2018).

Gómez, M., Atencio, E. y Muñoz, F., Competencias en management para la formación de ingenieros civiles, Revista Iberoamericana de Educación en Ingeniería (RIEI), 1(2), (2019).

Hebles, M. y Llanos-Contreras, O., Evolución percibida de la competencia para emprender a partir de la implementación de un programa de formación de competencias en emprendimiento e innovación, doi: 10.5944/reop.vol.30.num.1.2019.2519, REOP, 30, 9-26 (2019).

Hermosilla, P., Muñoz, F. y otros cuatro autores, A Proposal Of An Instrument To Evaluate Innovation Characteristics For Engineering Students, International Journal of Advanced Science and Technology, ISSN: 2005-4238, 29(11), 579590 (2020).

Herrera, R.F., Muñoz, F.C. y Salazar, L.A., Diagnóstico del trabajo en equipo en estudiantes de ingeniería en Chile, doi: 10.4067/S0718-50062017000500006, Form. Uni., 10(5), 49-61 (2017a).

Herrera, R.F., Muñoz, F.C. y Salazar, L.A., Perceptions of the development of teamwork competence in the training of undergraduate engineering students, Glob. J. Eng. Educ., 19(1), 30-35 (2017b).

Herrera, R., Vielma, J.C. y Muñoz, F., Impact of microteaching on engineering students' communication skills, Inter. J. Eng. Educ., 34(6), 1768-1775 (2018).

Jarrar, M. y Anis, H., The Impact of Entrepreneurship on Engineering Education, Proc. 2016 Canadian Engineering Education Association (CEEA16) Conf., 2-7 (2016).

Jiménez-Medina, E., Rojas-Arenas, I., Competencias Profesionales E Industria 4.0: Análisis Exploratorio para Ingeniería Industrial y Administrativa para el Área Metropolitana Del Valle De Aburrá. 2do Congr. Latinoaméricano Ing. (2019).

Liao, Y., Deschamps, F., De Freitas, E. y Pierin, L., Past, present and future of Industry 4.0 - a systematic literature review and research agenda proposal, doi: 10.1080/00207543.2017.1308576, Int. J. Prod., 55(12), 3609-3629 (2017).

Lounsbury, M., Cornelissen, J., Granqvist, N. y Grodal, S., Culture, innovation and entrepreneurship, doi: 10.1080/14479338.2018.1537716, Innovation, 1-12 (2018).

Mayhew, M.J., Simonoff, J.S., Baumol, W.J., Wiesenfeld, B.M. y Klein, M.W., Exploring Innovative Entrepreneurship and Its Ties to Higher Educational Experiences, doi: 10.1007/s11162-012-9258-3, Res. High. Educ., 53, 831-859 (2012).

Mohamed, M. Challenges and Benefits of Industry 4.0: An overview, Int. J. Supply Chain Manag, 5(3), 256-265 (2018).

Morton, S., Pencheon, D. y Squires, N., Sustainable Development Goals (SDGs), and their implementation, doi: 10.1093/bmb/ldx031, Br. Med. Bull., 124, 81-90 (2019).

Naciones Unidas, Transforming our world: the 2030 agenda for sustainable development (2015). 
Cruz, N., Measurement of Graduate Attributes as a Tool for Educational Improvement: Case study of the Civil Engineering program in the University of Costa Rica, Universidad de Costa Rica - Pensamiento Educativo, Revista de Investigación Educacional Latinoamericana, 54(2), 1-16 (2017).

Oesterreich, T.D. y Teuteberg, F., Computers in Industry Understanding the implications of digitization and automation in the context of Industry 4.0: A triangulation approach and elements of a research agenda for the construction industry, doi: 10.1016/j.compind.2016.09.006, Comput. Ind., 83, 121-139 (2016).

Osborn, D., Cutter, A. y Ullah, F., Understanding the Transformational Challenge for Developed Countries, Report of a study by stakeholder forum (2015).

Oswald, M.R., Integrating Innovation and Entrepreneurship Principles into the Civil Engineering Curriculum, doi: 10.1061/(ASCE)El.1943-5541.0000233, J. Prof. Issues Eng. Educ. Pract., 141(3), 1-8 (2015).

Passow, H. y Passow, C., What competencies should undergraduate engineering programs emphasize? A systematic review, doi: 10.1002/jee.20171, J. Eng. Edu., (2017).

Psacharopoulos, G. y Schlotter, M., Skills for Employability, Economic Growth and Innovation: Monitoring the Relevance of Education and Training Systems, European Expert Network on Economics of Education (2010)

Radharamanan, R., Juang, J., Innovation and entrepreneurship in engineering education at MUSE. Radharamanan, R., \& Juang, J.-N. (2012). Innovation and entrepreneurship in engineering education at MUSE. Journal of the Chinese Institute of Engineers, 35(1), 25-36. doi:10.1080/02533839.2012.624797. J. Chinese Inst. Eng., 37-41 (2012).

Rejikumar, G., Raja, V., y otros tres autores, Industry 4.0: key findings and analysis from the literature arena, doi: 10.1108/BIJ-09-2018-0281, Benchmarking: An International Journal, 26(8), 2514-2542 (2019).

Shuli, Z., Hua, Z., y Junlin, W., Cognition and System Construction of Civil Engineering Innovation and Entrepreneurship System in Emerging Engineering Education, doi: 10.1016/j.cogsys.2018.10.020, Cogn. Syst. Res., 52, 1020-1028 (2018).

Solesvik, M., Entrepreneurial competencies and intentions: The role of higher education, doi: 10.23762/FSO_VOL7_NO1_1, Journal Forum Scientiae Oeconomia, 7(1), 9-23 (2019).

Stock, T., Perspectives for International Engineering Education: between used capacity and operational efficiency, doi: 10.1016/j.promfg.2018.02.089, Procedia Manuf., 21, 10-17 (2018).

Sung, T. K., Industry 4.0: A Korea perspective, doi: 10.1016/j.techfore.2017.11.005, Technol. Forecast Soc. Change, 132, 40-45 (2018).

Toner, P., Workforce skills and innovation: an overview of major themes in the literature. Paris (2011).

Wiek, A., Withycombe, L. y Redman, C., Key competencies in sustainability: a reference framework for academic program development, doi: 10.1007/s11625-011-0132-6, Sustain. Sci., 6, 203-218 (2011).

Xu, L.D., Xu, E.L. y Li, L., Industry 4.0: state of the art and future trends, doi: 10.1080/00207543.2018.1444806, Int. J. Prod., 56(8), 2941-2962 (2018). 
